\title{
Research on the Dynamics of Crankshaft Under the Condition of Operating Mode Based on LS-DYNA
}

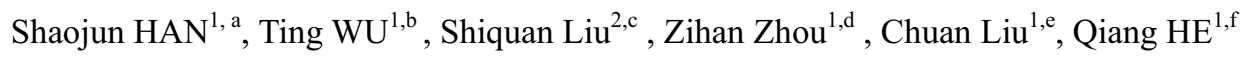 \\ ${ }^{1}$ School of Mechanical and Electronic Engineering, Wuhan University of Technology, Wuhan, China \\ ${ }^{2}$ Shanghai Automotive Gear Works, Shanghai, China \\ ahanshjun@163.com, bhbwuting@163.com, ${ }^{\mathrm{c}} 702057684 @$ qq.com, ${ }^{\mathrm{d}} 864426302 @$ qq.com, ${ }^{\mathrm{e}} 772837913 @$ @q.com, \\ fqianghe1992@163.com
}

\begin{abstract}
A model of crankshaft under working condition is established based on LS-DYNA. The stress distributions of crankshaft and its regularities of the stress along surface of crankshaft and layer depth at the fillet are studied. The results show that the obvious stress concentration exist in the upper end of fillet on main journal and the lower end of fillet on rod journal when the piston arrives at top dead center, and the maximum equivalent stress occurs at the fillet on rod journal, so the fracture of crankshaft is most likely to occur in the lower end of fillet on rod journal. With the increase of depth, the stress on the surface of fillet reduces correspondingly, and the equivalent stress begins to stabilize when the depth reaches up to $2.8 \mathrm{~mm}$. The study of maximum working stress provides the theory basis for the selection of residual stress.
\end{abstract}

Keywords: crankshaft; explicit dynamics; distribution regularities; residual stress

\section{Introduction}

Crankshaft is one of the most important parts of the engine. Due to the impact of cyclic stress, the permanent deformation will occur at the crankshaft ${ }^{[1]}$. How to ensure the crankshaft working life and prevent the occurring of fracture has been the research topic over the years. In order to study the stress distribution of crankshaft under the working condition, this paper builds and analyzes the model of crankshaft based on LS-DYNA, and gets the results of the stress distribution and the regularities of the stress along the fillet surface and layer depth in fillet of connecting rod journal.

The crankshaft fillet rolling strengthening is an ideal surface strengthening method. Compared with the traditional methods, rolling strengthening has many advantages like low cost, short processing time, obvious strengthening effect ${ }^{[2]}$ etc. After rolling strengthening, the crankshaft fatigue strength can increase by $30 \%-200 \%{ }^{[3]}$.
However, how much the residual stress produced in the process of rolling strengthening is uncertain, and it's very hard to confirm the value of residual stress. This paper finds that the maximum working stress can provide the theory basis for the selection of residual stress.

\section{Research of kinematics of crankshaft connecting rod system and load-bearing performance}

\subsection{Kinematics analysis of crankshaft connecting rod system}

Figure 1 shows the structural diagram of crankshaft connecting rod. $\alpha=0^{0}$, the piston moves to the top dead center position. $\alpha=180^{\circ}$, the piston moves to the lower dead center position. 


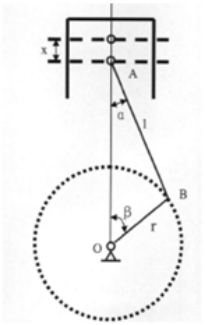

Fig.1 Structural diagram of crankshaft connecting rod

If $\lambda=l / r, \sin \alpha=\lambda \sin \beta$, the displacement formula is shown below

$$
x=r\left[(1+1 / \lambda)-\cos \beta-\left[1-\lambda^{2} \sin ^{2} \beta\right] / \lambda\right] .
$$

According to the piston displacement formula, it deduces the velocity and acceleration:

$$
\begin{gathered}
v=w r\left[\sin \beta+\lambda / 2 \sin 2 \beta\left[1-\lambda^{2} \sin ^{2} \beta\right]^{-\frac{1}{2}}\right] . \quad(2) \\
a=w^{2} r\left[\cos \beta+\lambda\left(1-\lambda^{2} \sin \beta\right)^{-3 / 2}\left[\cos 2 \beta\left(1-\lambda^{2} \sin \beta\right)^{2}+\frac{\lambda^{2}}{4} \sin ^{2} 2 \beta\right]\right] .
\end{gathered}
$$

\subsection{Gas force and inertia force of connecting rod mechanism}

The force of the cylinder is not only related to the size of the cylinder, but also to the pressure between inside and outside of the cylinder. The gas force formula is shown as follows:

$$
F_{g}=\left(P_{1}-P_{0}\right) \pi D^{2} / 4 \cos \alpha \text {. }
$$

$\alpha$ :swing angle of connecting rod; $D$ :cylinder diameter; $P_{1}$ :absolute pressure of gas in cylinder; $P_{0}$ :absolute pressure of gas inside the box.

In the theoretical analysis, the whole weight of connecting rod is translated into large mass $m_{1}$ and small mass $m_{2}$ by the static equivalent principle, $m_{1}$ is heavier than $m_{2}$. Reciprocating inertia force is generated by the piston and small mass. It is considered that the weight of piston assembly concentrates on the center of the pin. $M_{p}=\sum m_{i}$. The reciprocating inertia force is shown as follows:

$F_{1}=\left(M_{p}+m_{2}\right) r w^{2}\left[\cos \beta+\lambda\left(1-\lambda^{2} \sin \beta\right)^{-3 / 2}\left[\cos 2 \beta\left(1-\lambda^{2} \sin \beta\right)^{2}+\frac{\lambda^{2}}{4} \sin ^{2} 2 \beta\right]\right]$

Centrifugal inertia force is generated by the crankshaft and the large mass of connecting rod. The crankshaft comprises the main crankshaft journal, crank arm, connecting rod journal etc. In order to facilitate the theoretical calculation, the crankshaft's weight is converted to the connecting rod's weight, so the centrifugal inertia force after converting weight is equal to the centrifugal inertia force before converting. The single crankshaft's weight conversion formula is shown as follows:

$m_{k}=m_{a}+2 m_{b} p / x$.

$m_{a}$ : weight of connecting rod journal; $m_{b}$ : weight of crank arm; $p$ : distance between the center of crank arm and rotating center of crankshaft; $x$ : piston stroke. The centrifugal inertia force is shown as follows:

$$
F_{2}=\left(m_{k}+m_{1}\right) r w^{2} \text {. }
$$

\subsection{Solution of load}

There're some parameters of crankshaft showed in table 1.

Table 1 Parameters of the crankshaft

\begin{tabular}{|c|c|c|c|}
\hline Parameters & Value & Parameters & Value \\
\hline crank radius $\boldsymbol{r}$ & $39 \mathrm{~mm}$ & $\begin{array}{c}\text { Bulk mass } \\
\text { quality } \boldsymbol{m}_{\mathbf{1}}\end{array}$ & $0.342 \mathrm{~kg}$ \\
\hline Connecting rod & $130 \mathrm{~mm}$ & $\begin{array}{c}\text { head mass } \\
\text { quality } \boldsymbol{m}_{\mathbf{2}}\end{array}$ & $0.180 \mathrm{~kg}$ \\
center distance $\boldsymbol{l}$ & & single crank & $0.281 \mathrm{~kg}$ \\
\hline main journal & $50.33 \mathrm{~mm}$ & quality $\boldsymbol{m}_{\boldsymbol{k}}$ & \\
\hline diameter $\boldsymbol{D}_{\boldsymbol{1}}$ & & piston quality & $0.315 \mathrm{~kg}$ \\
connecting rod & $38.5 \mathrm{~mm}$ & $\boldsymbol{\boldsymbol { m } _ { \boldsymbol { p } }}$ & \\
\hline diameter $\boldsymbol{d}$ & & angular velocity & $255.2 \mathrm{rad} / \mathrm{s}$ \\
cylinder diameter & $75 \mathrm{~mm}$ & $\boldsymbol{w}$ & \\
\hline $\boldsymbol{D}$ & & &
\end{tabular}

This paper finds out the similar cylinder size and the size of the cylinder pressure, the maximum pressure in the $465 \mathrm{Q}$ cylinder is about $8.12 \mathrm{MPa}$, the inner pressure of the box is about $0.12 \mathrm{MPa}$.

$$
\begin{aligned}
& F_{g}=\left(P_{1}-P_{n}\right) \pi D^{2} / 4 \cos \alpha=35325 N \\
& F_{1}=\left(M_{p}+m_{2}\right) a=1634 N \\
& F_{2}=\left(m_{k}+m_{1}\right) r w^{2}=1582 N
\end{aligned}
$$

During the power stroke, the maximum pressure of crankshaft is shown as follows:

$$
F=F_{g}-F_{1}-F_{2}=32109 N \text {. }
$$

\section{Dynamics analysis of the crankshaft under working condition}

\subsection{Parameter selection}

The diameter of the main journal is $50.33 \mathrm{~mm}$, the diameter of the connecting rod journal is $38.5 \mathrm{~mm}$, the diameter of the cylinder is $75 \mathrm{~mm}$. Material of crankshaft is made of QT800 ductile iron, its density is $7.16 \times$ $10^{3} \mathrm{~kg} / \mathrm{m}^{3} \quad$, modulus of elasticity is $7.12 \times 10^{5} \mathrm{Mpa} / \mathrm{mm}^{3}$, the poisson's ratio is 0.27 . The 
actual stress-strain curve of ductile cast iron is shown in figure 2. Connecting rod is made of $42 \mathrm{CrMo}$, its density is $7.85 \times 10^{3} \mathrm{~kg} / \mathrm{m}^{3}$, modulus of elasticity is $2.09 \times$ $10^{5} \mathrm{MPa} / \mathrm{mm}^{3}$, the poisson's ratio is 0.28 . Piston pin is usually made of $\mathrm{T} 10$, its density is $7.86 \times 10^{3} \mathrm{~kg} / \mathrm{m}^{3}$, modulus of elasticity is $2.08 \times 10^{5} \mathrm{MPa} / \mathrm{mm}^{3}$, the poisson's ratio is 0.28 .

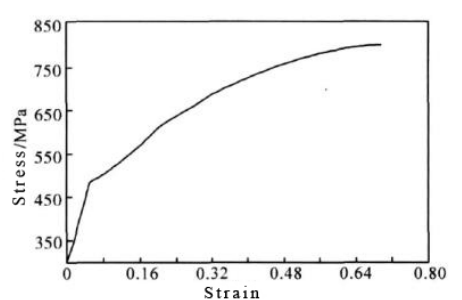

Figure.2 Stress-strain curve of ductile cast iron

\subsection{Simplified simulation model}

This paper makes an appropriate simplification of the crankshaft structure and takes a part of the crankshaft as the calculating object. The crankshaft fatigue point occurs in the third crank throw of connecting rod journal, so this paper chooses the third crank throw as the analysis model. The simplified model is composed of half single crank throw, connecting rod and piston pin. The simplified model is shown in figure $3 \mathrm{a}$, and its element type is SOLID164 unit, it is shown in figure $3 b$.

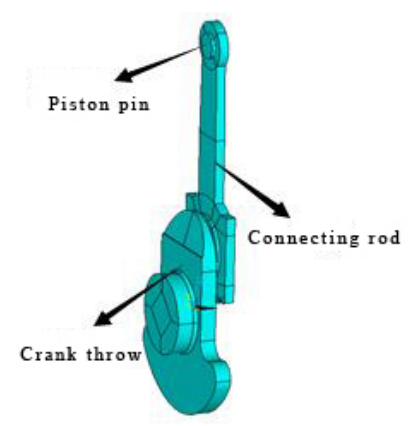

Figure.3a Solid model

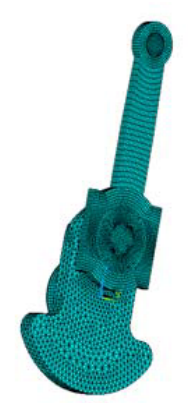

Figure.3b SOLID164 unit

\subsection{Apply Load}

During the process of working, the impact load of the crankshaft is maximum when it's in the stage of power stroke which is the key point of leading to the fatigue of the crankshaft. This paper mainly analyzes the crankshaft when it's in the stage of power stroke.

The normal speed of the crankshaft is around 1500 3000r/min, this paper takes the constant speed $2400 \mathrm{r} / \mathrm{min}$ for analysis speed. The load value is $\mathrm{F}_{\max } / 2$. The loading force is not constant, when the gas bursts, the pressure in the cylinder increases rapidly to the maximum. As the piston moves down, the gas volume increases and the pressure begins to fall.

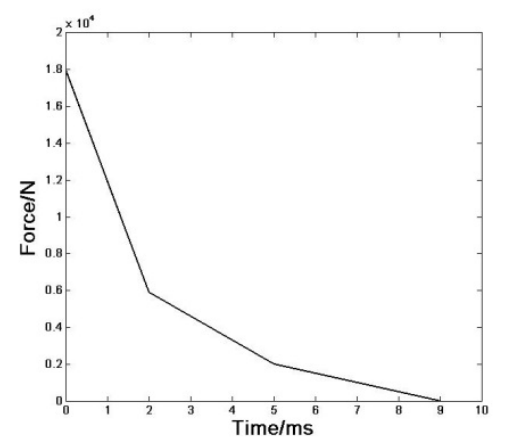

Figure.4 Force-time curve

In order to make the load and the actual gas pressure closer, the force-time curve is established in figure 4. With the increase of rotation angle of crankshaft, the load decreases rapidly. When the time is $0.009 \mathrm{~s}$, the load decreases to 0 . The terminal time in this paper is $0.009 \mathrm{~s}$.

\subsection{Solution and result processing}

Figure 5a shows the equivalent stress of the main journal and figure $5 \mathrm{~b}$ shows the equivalent stress of the connecting rod journal in the maximum load. From the simulation results, it can be seen that the upper end of the main journal and the lower end of the connecting rod journal exist obvious stress concentration phenomenon, and the maximum equivalent stress, $901 \mathrm{MPa}$, occurs at the fillet of the connecting rod journal. 


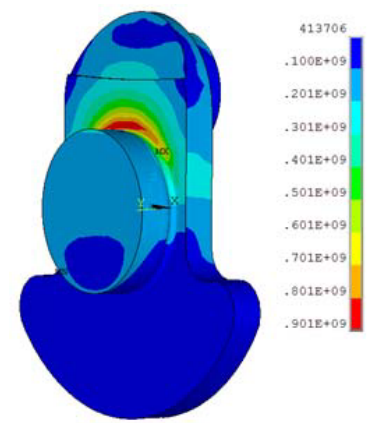

Figure.5a Equivalent stress of main journal

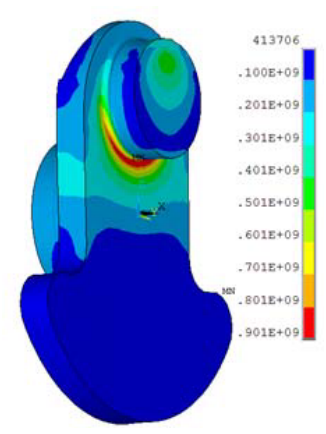

Figure.5b Equivalent stress of connecting rod journal

Analysis result shows that the upper end of the main journal withstands the pressure stress and the lower end of the connecting rod journal withstands the tensile stress. Rigid material can withstand the more pressure stress than tensile stress. Therefore, tensile stress in the fillet of the connecting rod journal is the key factor of leading to fatigue failure. This paper analyzes the tensile stress in the fillet of the connecting rod journal. In order to analyze it intuitively, the two paths in the circumferential direction and the rolling direction of the fillet of the connecting rod journal are selected. The paths in two directions are shown in Figure 6.

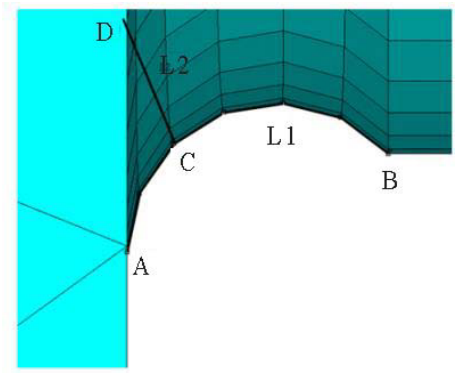

Figure.6 Paths in two directions

The path $\mathrm{L} 1$ is from $\mathrm{A}$ to $\mathrm{B}$ in the connecting rod journal along the circumferential direction, the path L2 is from $\mathrm{C}$ to $\mathrm{D}$ in the connecting rod journal along the rolling direction. The equivalent stress values and the stress distributions along path L1 and path L2 are obtained from the simulation which is shown in Figure $7 \mathrm{a}$ and $7 \mathrm{~b}$. In Figure 7a, it can be seen a parabolic form of the fillet surface equivalent stress curve, the maximum value of equivalent stress in the fillet of connecting rod journal is $888 \mathrm{MPa}$ and the point of maximum equivalent stress locates in between A and C. In Figure 7b, it can be seen that the stress in the fillet of the connecting rod journal gradually reduces with the increase of the depth, when the depth approaches $2.8 \mathrm{~mm}$, the equivalent stress is beginning to stabilize, and the maximum equivalent stress on the surface is $860 \mathrm{MPa}$.

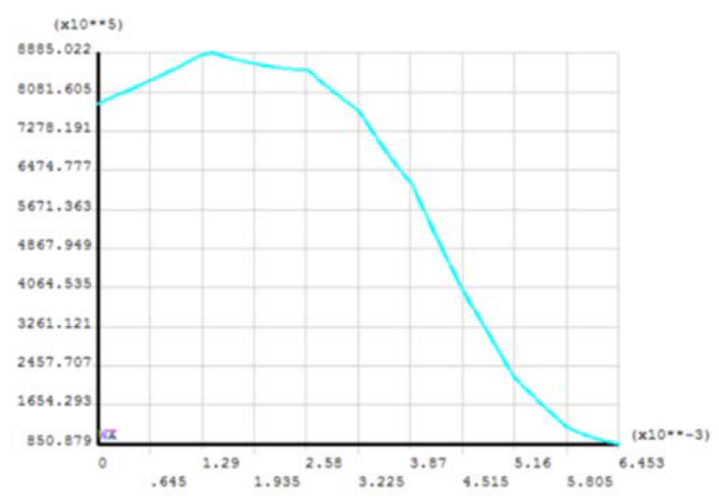

Figure.7a Simulation result of path L1

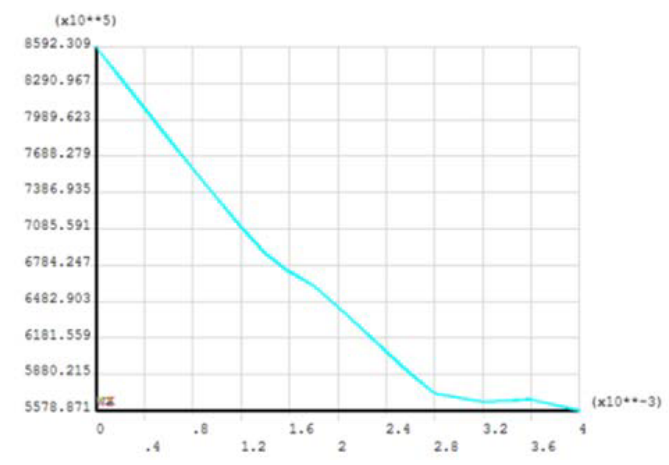

Figure.7b Simulation result of path L2

\section{Summary}

1. This paper researches the maximum equivalent stress, and gets the stress concentration phenomenon in the lower end of the connecting rod journal and the upper end of the main journal. The maximum equivalent stress occurs at the fillet of the connecting rod. Therefore, the key factor of leading to fatigue failure is the stress in the fillet of the main journal and the connecting rod.

2. The stress distributions along the path L1 and the path L 2 are obtained by simulation. They can provide the theory basis for the selection of the depth of the carburizing layer. 
3. The maximum working stress, $901 \mathrm{MPa}$, is obtained by simulation. It can provide the theory basis for the selection of the residual stress in the process of crankshaft fillet rolling.

\section{References}

1. Chen Lidong, Ma Shuying, Liu Rongchang, Li Shuzhen, Shi Lei, Li Guofang, Zheng Lixin. Design of hydraulic system of crankshaft rolling strengthen machine, Machine Tool and Hydraulics, 2009,7(37): 66-68

2. Xue Longquan, Wang Yuqiu, Liu Rongchang, Zhang Hongjun, Wang Huiwu. Finite element analysis of residual deforming of crankshaft rolling process, Machine Tool and Hydraulics, 2005,10: 40-41

3. Wang Huiwu, Xue Longquan, Liu Hongzhao, Zhou Chunguo, Liu Rongchang. The design of eccentric fixture of crank for fillet rolling, Machine Tool and Hydraulics,2004,8:164-165

4. Liu Rongchang, Sun Huadong, Jiao Honglei, Ma Shuying, Xue Longquan. Explicit dynamic analysis of crankshaft rolling process, Transactions of Csice,2009,5(27):463-468 